\title{
DIVERSITY OF PLANT PARASITIC NEMATODES ASSOCIATED WITH SOME VEGETABLES IN SINAI PENINSULA, EGYPT
}

\author{
El-Nuby, Ahmed S.M. ${ }^{1^{*}}$, Sayed A. Montasser $^{2}$ and Ismail A. El- \\ Khadrawy ${ }^{1}$ \\ ${ }^{1}$ Department of Plant Protection, Desert Research Center, El-Matareya, \\ Cairo, Egypt \\ ${ }^{2}$ Department of Zoology and Nematology, Faculty of Agriculture, Al- \\ Azhar University, Cairo, Egypt \\ *E-mail: ahmedelnuby.drc@gmail.com
}

survey for plant parasitic nematode (PPN) fauna in Sinai A Peninsula, Egypt was carried out. A total of 607 soil and root samples was collected, at growth seasons during the period from 2016 to 2017 at both governorates; North Sinai (in which few surveys were accomplished) and South Sinai for the first time. The nematode survey was based on collecting soil and root samples around the roots. In the two governorates, 13 genera in 9 families, belonging to 3 orders of phylum Nematoda were observed. These orders were Dorylaimida, Triplonchida and Tylenchida. Three genera viz., Meloidogyne, Rotylenchulus and Tylenchorhynchus belonging to order Tylenchida were found to represent the highest plentiful numbers (18891, 3528 and 3316, respectively) in Sinai Peninsula. Meloidogyne was the most abundant and dominant genus in all surveyed districts. Nematodes in North Sinai were more diverse than South Sinai, since they possessed 3 orders, 8 families and 13 genera (Belonolaimus, Criconema, Criconemoides, Helicotylenchus, Hoplolaimus, Meloidogyne, Pratylenchus, Rotylenchulus, Tetylenchus, Trichodorus, Tylenchorhynchus, Tylenchus and Xiphinema) versus 2 orders, 5 families and 6 genera viz., Criconema, Meloidogyne, Pratylenchus, Rotylenchulus, Trichodorus and Tylenchorhynchus in South Sinai. Species diversity peaked in Rafah district, whereas in South Sinai, peak was recorded in Ras-Suder district. Reviewing the previous investigations carried out in Sinai for recording plant parasitic nematode, it was found that the last 6 genera recovered from South Sinai were the first records, based on the present survey is considered the first nematological study carried out in this governorate. Also, this study will pay the growers attention, to control infestation by PPN.

Keywords: diversity, plant parasitic nematodes, survey, vegetables, Sinai Peninsula

The $1^{\text {st }}$ Conference of Plant Protection Science Applications for Sustainable Development of Desert Areas "Effect of Climate Change on Plant Pests and Biodiversity in Desert Environment" 19-20 October, 2019, Cairo-Egypt. 
Plant parasitic nematodes (PPN) are considered the main constrain for many crops, especially vegetables. Losses due to PPN are estimated at USD $\$ 130$ billion without considering other losses indirectly by interactions with other pathogens, also they are higher in developed countries compared to that of developing ones (Shakeel et al., 2012 and Becker 2014). Out of all PPN, root-knot nematodes (RKN), Meloidogyne have been reported as the most highly distributed ones all over the world and found in all terrains of all ecosystems, and in any soils have temperature more than $3^{\circ} \mathrm{C}$. They are considered the most economically important group of phytoparasitic nematodes due to their severe damage occurred for plants, particularly to vegetable crops and for their small-holder growers (Ibrahim et al., 2000; Ibrahim et al., 2010; Anwar and Mckenry, 2012; Shakeel et al., 2012; Adamou et al., 2013a and b; Korayem et al., 2014; Singh and Khanna, 2015; Kumar et al., 2017; Tariq-Khan, 2017 and Vindhyarani, 2017).

Vegetable crops are widely cultivated in Egypt and are common, which highly consumed by the peoples in all countries. Furthermore, small holder farmers in Sinai Peninsula depend on these crops production for consumption and improving their income.

Diversity of nematode fauna in agroecosystems and their relationships to soil processes were studied by Yeates and Bongers (1999), they used the soil nematode diversity as biomarkers for ecosystem. They suggested that nematodes were potential bioindicators and they found that changes in nematode diversity shown by values of the Shannon-Wiener index $\left(\mathrm{H}^{\prime}\right)$ often reflect environmental differences. They concluded that morphologically distinct groups or families may be useful within regions or soil types but fail to provide a universal indicator. Previous investigations in Egypt have shown the presence of about 54 genera and 160 species of PPN associated with different plants (Oteifa et al., 1997; Ibrahim et al., 2000; Ibrahim and El-Sharkawy, 2001 and Ibrahim et al., 2010). The association of PPN with vegetables was confirmed in Northern Egypt by other authors (Mokbel et al., 2006) as they found 9 genera of PPN in vegetable fields.

Although Sinai Peninsula has a strategic importance, there is little information about distribution of PPN associated with vegetable crops in this area, so the necessity of knowing phytonematodes diversity become urgent, in particular after selecting it as a part of national project for cultivation 1.5 million feddans. Few investigations were carried out in Sinai (confined in North Sinai only) as two surveys were accomplished by Ashoub (2010) and Korayem et al. (2014). However, successful production of vegetables in Sinai has been retarded by nematodes, particularly root-gall nematodes (RGN). Information concerning the occurrence and distribution of plant- parasitic nematodes in Egypt is important to assess their potential to cause economic damage to plants.

The objectives of this study were; identifying plant parasitic nematodes (PPN) associated with vegetable plants in Sinai Peninsula (Egypt),

Egyptian J. Desert Res., 69, Special Issue, 77-100 (2019) 
determination the frequencies of occurrence and population densities of nematodes in relation to locality and host species, using the diversity indices for measuring their distribution patterns in vegetable cultivation in Sinai. Documentation the presence and abundance of dominant genus or genera was illustrated in GPS- based map.

\section{MATERIALS AND METHODS}

\section{Sampling}

Nematological surveys were done in Sinai Peninsula, which including two governorates viz., North and South Sinai, besides Sahl El-Teina region (which irrigated from El-Salam canal) during 2016 to 2017. A total of 607 soil and root samples of vegetable crops were collected from the rhizosphere region at a depth of $15-30 \mathrm{~cm}$. Samples were randomly collected for each crop by making a zig-zag pattern across each field with soil auger at $20-\mathrm{cm}$ approximately depth. For plots up to 10 square meters in area, 2-3 sub-samples (cores) were taken per composite sample, the soil was then bulked in plastic bags, mixed carefully, and about $1000 \mathrm{~g}$ for each sample was placed in an ice box during collection and was transported, then stored at $4^{\circ} \mathrm{C}$ until processed in the lab. All samples were taken during the cropping growth season, March to October.

\section{Nematode Extraction and Identification}

Each soil sample was thoroughly mixed by shaking the plastic bags, then an aliqot of $250 \mathrm{~cm}^{3}$ sub-sample was used for extraction PPN genera using sieves (Sieve series of 100, 200 and 325 mesh-US Standard with openings of 149, 74 and $45 \mu \mathrm{m}$, respectively) following Cobb sifting and gravity method (Cobb, 1918). Light microscope was used for identification and enumeration of nematodes. Identification of nematodes genera, which fixed in $2 \%$ hot formaldehyde solution by binocular microscope, was based on the morphological characters of adult and juvenile forms according to different references (Goodey, 1963; Tarjan, 1973; Mai and Lyon, 1975; Anderson and Mulvey 1979 and Mekete et al., 2012)

\section{Nematode Estimation}

Data registrations were based on two parameters viz. frequencies of occurrence (FO) and population densities (PD) per $250 \mathrm{~cm}^{3}$ soil. The $\mathrm{FO} \%$ of the PPN was determined from the relationship among the numbers of samples (e) in which the genus was found divided by the total number of samples taken (E) from that location or crop, multiplied by 100 to express as a percentage $[(\mathrm{FO}=\mathrm{e} / \mathrm{E}) \mathrm{X} 100]$ according to (Sawadogo et al., 2009). PD of nematode species was calculated as the averages of the total number of nematodes recorded for those samples in which a nematode species was found 
(summation of individuals of specific nematode genus in all samples/total number of samples containing the same genus) as described by Norton (1978).

\section{Diversity Indices}

To evaluate the diversity, three biological indices were used (e.g. Shannon-Wiener index, evenness index, and the Simpson dominance index). Typically, Shannon's ( $\left.H^{\prime}\right)$ index is sensitive to rare taxa, however Simpson's $\lambda$ index weights common taxa (Boyle et al., 1990), the utilization of richness, evenness and diversity indices cannot be separated. Evenness indices can be considered as relative diversity indices or normalizations of diversity indices (Peet, 1974 and Kvalseth, 1991). In addition, species richness (S) or species abundance represents the simplest biodiversity index and it does not consider any characteristic of taxon or their relative abundance.

Taxonomical diversity of phytoparasitic nematode communities was estimated by: (a) the total number of nematode in each district $(\mathrm{N})$; (b) the species richness (S) represent the total number of taxa in a community; (c) the Shannon-Wiener diversity index $\left(\mathrm{H}^{\prime}\right)$ according to Shannon and Wiener (1949) using the formula: $H^{\prime}=-\Sigma[(\mathrm{pi}) \times 1 \mathrm{n}$ (pi)], where pi is the proportion of individuals $(\mathrm{n} / \mathrm{N})$ in each species, $\mathrm{i}$ that counted the local diversity $\left[\mathrm{H}^{\prime}\right.$ ranged from 0 to $\ln (\mathrm{S})$ ], $\mathrm{H}$ max= the maximum of Shannon index (Maximum diversity possible); (d) the Simpson index (Simpson, 1949) or dominance index (D) and can actually refer to any one of 3 closely related indices. There are two versions of the formula for calculating using formula, either of them is acceptable;

$\mathrm{D}=\Sigma(\mathrm{Pi})^{2}$ or $\Sigma(\mathrm{n} / \mathrm{N})^{2}$ and $\Sigma \mathrm{n}(\mathrm{n}-1) / \mathrm{N}(\mathrm{N}-1)$, the last were used, the value of $\mathrm{D}$ ranges between 0 and 1 . With this index, 0 represents infinite diversity and 1 , no diversity, i.e. the bigger the value of $\mathrm{D}$, the lower the diversity. This is neither intuitive nor logical, so to overcome these problem, two modifications in D can be used: first; (1-D) the value of this index also ranges between 0 and 1 , but now, the greater the value, the greater the sample diversity, secede; (1/D) the higher the value of this index means that greater diversity are found, also the maximum value is the number of taxa. and (e) the evenness or equity index that are considered also as relative diversity indices or normalizations of diversity indices that assess the uniformity of taxa distribution within the community, value of $\mathrm{E}$ can be calculated according to Pielou (1975) by this formula;

$\mathrm{E}=\mathrm{H}^{\prime} / \mathrm{Hmax}=\mathrm{H}^{\prime} / \operatorname{lnS}, \ln (\mathrm{S})$ i.e. natural logarithm of total number of taxa, species, found (E ranged between 0 and 1).

\section{RESULTS}

Survey results of phytoparasitic nematodes associated with different vegetable crops in different districts of Sinai are shown in (Tables 1-4). Data in table (1) reveal that 13 genera of stylet-bearing nematodes (Belonolaimus,

Egyptian J. Desert Res., 69, Special Issue, 77-100 (2019) 
Criconema, Criconemoides, Helicotylenchus, Hoplolaimus, Meloidogyne, Pratylenchus, Rotylenchulus, Tetylenchus, Trichodorus, Tylenchorhynchus, Tylenchus and Xiphinema) were isolated from 404 samples collected from North Sinai governorate. Meloidogyne was more frequent in all districts than other genera, followed by Tylenchorhynchus, which was found in five districts. FO of Meloidogyne peaked in Rafah and El-Sheikh Zuweid (77.9 and $65.1 \%$, respectively). The maximum PD of Meloidogyne was present in El-Arish and El-Tina plain (3400 and 3050 individuals $/ 250 \mathrm{~cm}^{3}$ soil, respectively).

The diversity of PPN was greater in Rafah district compared with others. Belonolaimus and Xiphinema were confined to Rafah district only and were not observed in any vegetable fields, and their frequencies were the lowest compared to other PPN genera in Rafah district as also were found in all district in North Sinai government, besides Criconema and Tylenchus $(0.25 \%)$. El- Hassana district was the lowest one in the nematode diversity, since it had one Meloidogyne. Tetylenchus recovered once from Al Arish is similar to Tylenchus from El-Tina plain. Collective data of North Sinai revealed that Meloidogyne was the most abundant genus (44.8\%) and also possessed the maximum population density (1330), followed by Rotylenchulus (7\%), Trichodorus (3.5\%), Tylenchorhynchus (2.7\%), Helicotylenchus, Hoplolaimus and Pratylenchus (2.5\%) and Criconemoides $(1.5 \%)$, whereas the minimum frequent genera were Belonolaimus, Criconema, Tetylenchus, Tylenchus and Xiphinema (0.25\%).

From South Sinai governorate, a total of 203 samples was collected from vegetable crops, of which six PPN genera were found (Criconema, Meloidogyne, Pratylenchus, Rotylenchulus, Trichodorus and Tylenchorhynchus) as presented in table (2). Meloidogyne and Rotylenchulus were found in all districts. The highest FO in all governorates was recorded by Meloidogyne (34.5\%), followed by Rotylenchulus (10.3\%) and Tylenchorhynchus (7.4\%), whereas Criconema and Pratylenchus were the least frequent genera $(0.5 \%)$. Nuweiba and Ras Suder included more nematode genera than other districts. Similar to North Sinai, the most dominant genus was Meloidogyne; its highest FO of 72.0 and $47.0 \%$ were recorded in Ras Suder and Abo Zenima, respectively. Whereas the lowest frequency (16.2\%) was observed in Saint Katherine. Meloidogyne was found in greater population densities in El-Tour and Nuweiba (1848 and 1125 individuals, respectively). Rotylenchulus was recorded in maximum frequency at El-Tour (33.3\%), however density peaked in Saint Katherine (700 individuals). Tylenchorhynchus was more frequent than the rest recovered genera, since it was recorded in three districts.

Results listed in table (3) show that PPN community in North Sinai varied according to the vegetable hosts. It was observed that favorable hosts of Solanaceae plants were tomatoes and eggplants. It was noticed that tomato plants harbored the maximum number of nematode genera (12) followed by 
eggplant (8), however pepper possessed the lowest number of genera (4). The highest PO and FO of Meloidogyne were observed in eggplant rhizosphere (2265 individuals $/ 250 \mathrm{~cm}^{3}$ soil and $55.6 \%$, respectively), followed by tomato (825 individuals and $37.3 \%$ ), the pepper came in the last category (806 individuals and 34.3\%). Within Cucurbitaceae plants; cucumber and watermelon harbored the highest number of nematode genera (8), followed by squash (6), whereas cantaloupe possessed the lowest number of genera (4). Meloidogyne genus peaked in its frequency with watermelon, followed by cucumber (61.9 and $57.1 \%$, respectively), but the maximum density was gained by cantaloupe (1333 individuals $/ 250 \mathrm{~cm}^{3}$ soil). It was noticed that sedentary endo and semi endo-parasitic genera; Meloidogyne and Rotylenchulus, respectively, were found in all vegetable hosts of both families (Solanaceae and Cucurbitaceae).

Data presented in table (4) show that PPN fauna in South Sinai were different from host plant to another. Tomatoes were bearing four PPN genera, Meloidogyne was found at high frequency (37.3\%) and density (1374 individuals), compared to other PPN genera. Cucumber harbored the maximum density (1163 individuals) and frequency (44.4\%) of Meloidogyne, also cucumber bore the highest density of Tylenchorhynchus $(6600$ individuals). The last genus was recorded in all vegetable hosts, expect cantaloupe, whereas Meloidogyne came secondly. Squash recorded the lowest densities, especially for Meloidogyne and Rotylenchulus (200 individuals), but the frequency was the highest with Meloidogyne (50\%) and equal in Rotylenchulus, Trichodorus and Tylenchorhynchus (25\%). Watermelon associated with only Tylenchorhynchus, achieving the maximum frequency $(50 \%)$.

Data in table (5) reveal that morphological and morphometric studies of diagnostic characters confirmed the identification of 3 orders, 8 families and 13 genera of PPN (Belonolaimus, Criconema, Criconemoides, Helicotylenchus, Hoplolaimus, Meloidogyne, Pratylenchus, Rotylenchulus, Tetylenchus, Trichodorus, Tylenchorhynchus, Tylenchus and Xiphinema) associated with vegetable crop soils in Sinai Peninsula. Among them rootknot, reniform and stunt nematodes were the most abundant.

Egyptian J. Desert Res., 69, Special Issue, 77-100 (2019) 


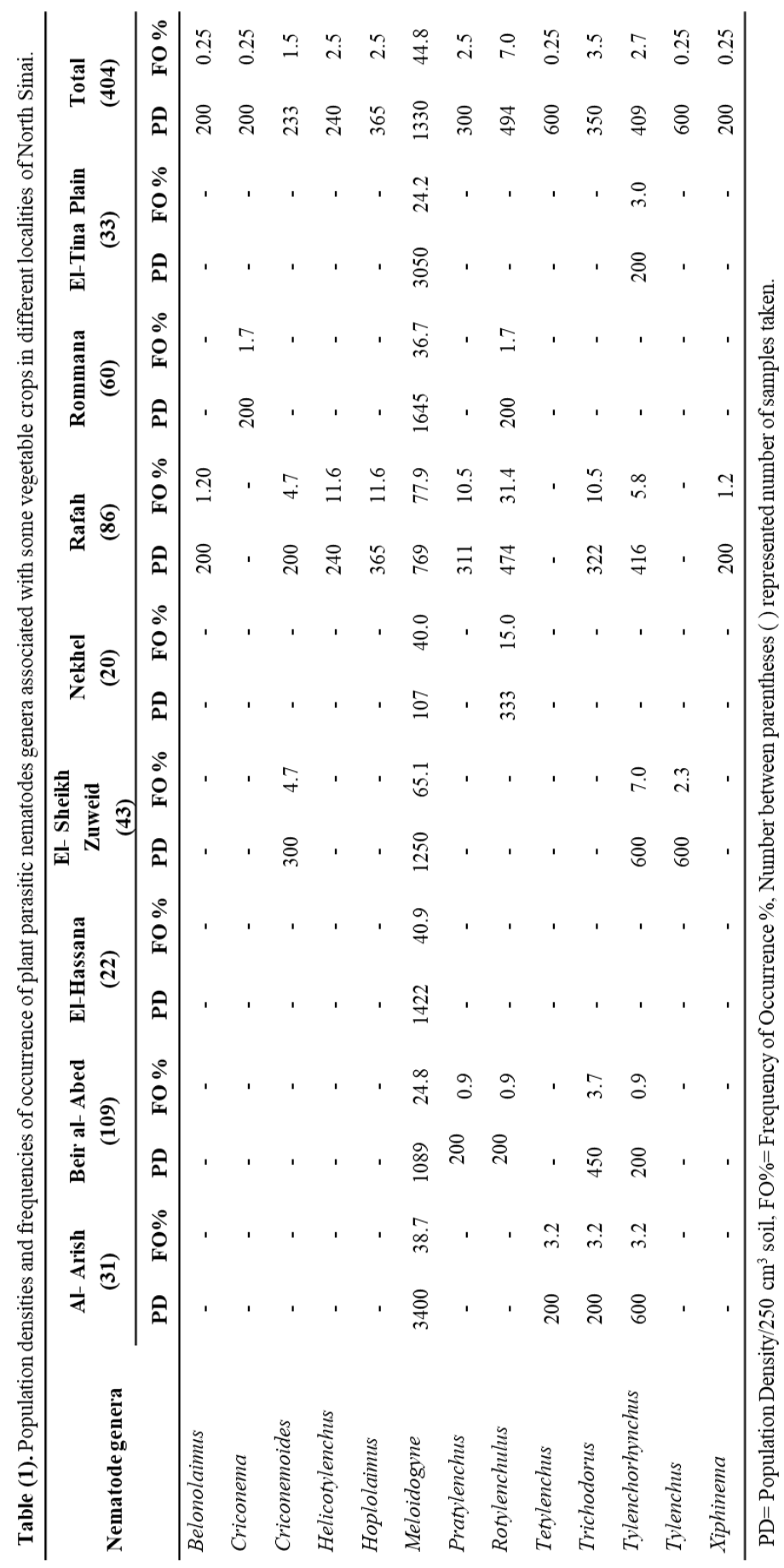

Egyptian J. Desert Res., 69, Special Issue, 77-100 (2019) 


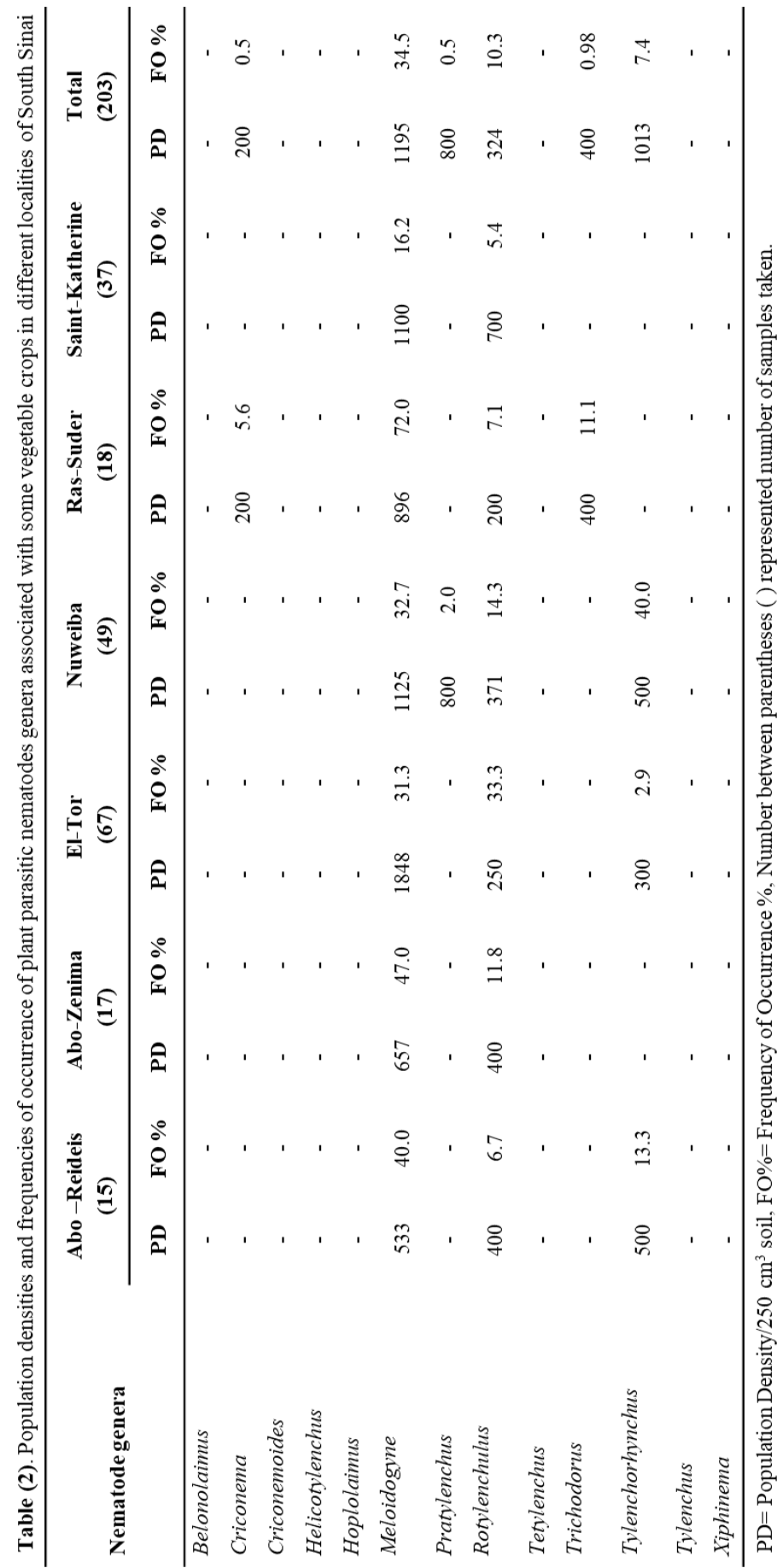

Egyptian J. Desert Res., 69, Special Issue, 77-100 (2019) 


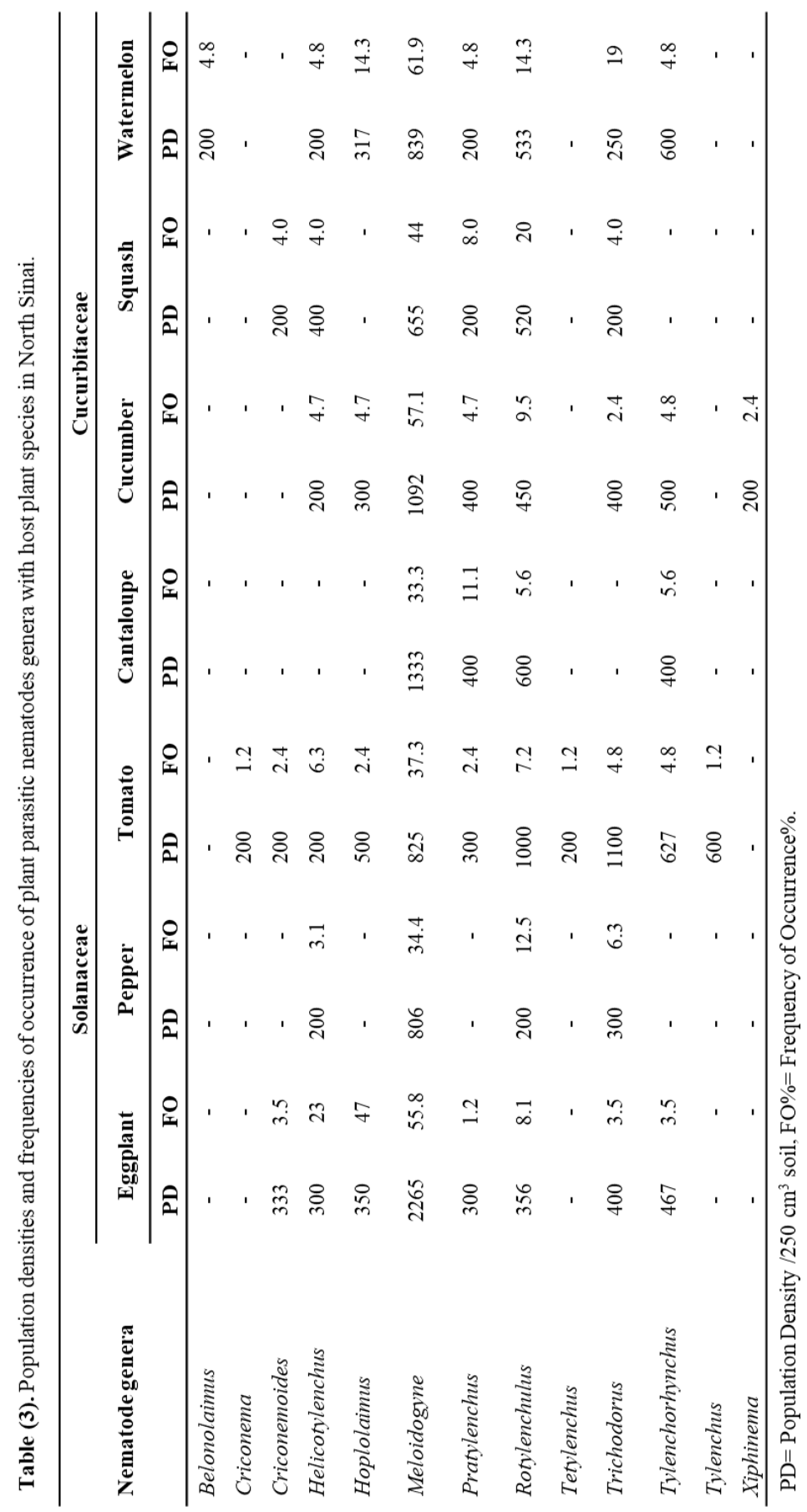

Egyptian J. Desert Res., 69, Special Issue, 77-100 (2019) 


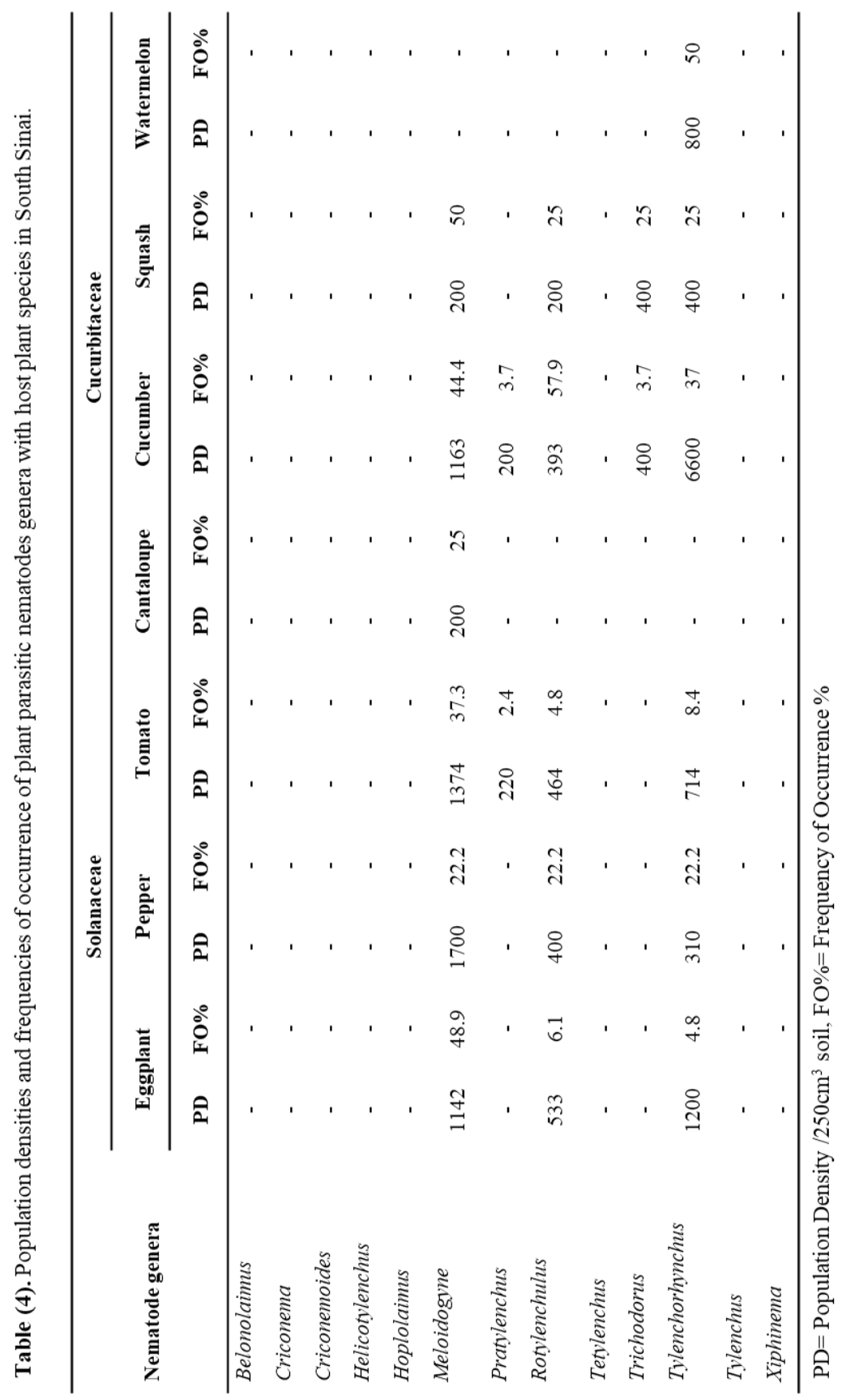

Egyptian J. Desert Res., 69, Special Issue, 77-100 (2019) 


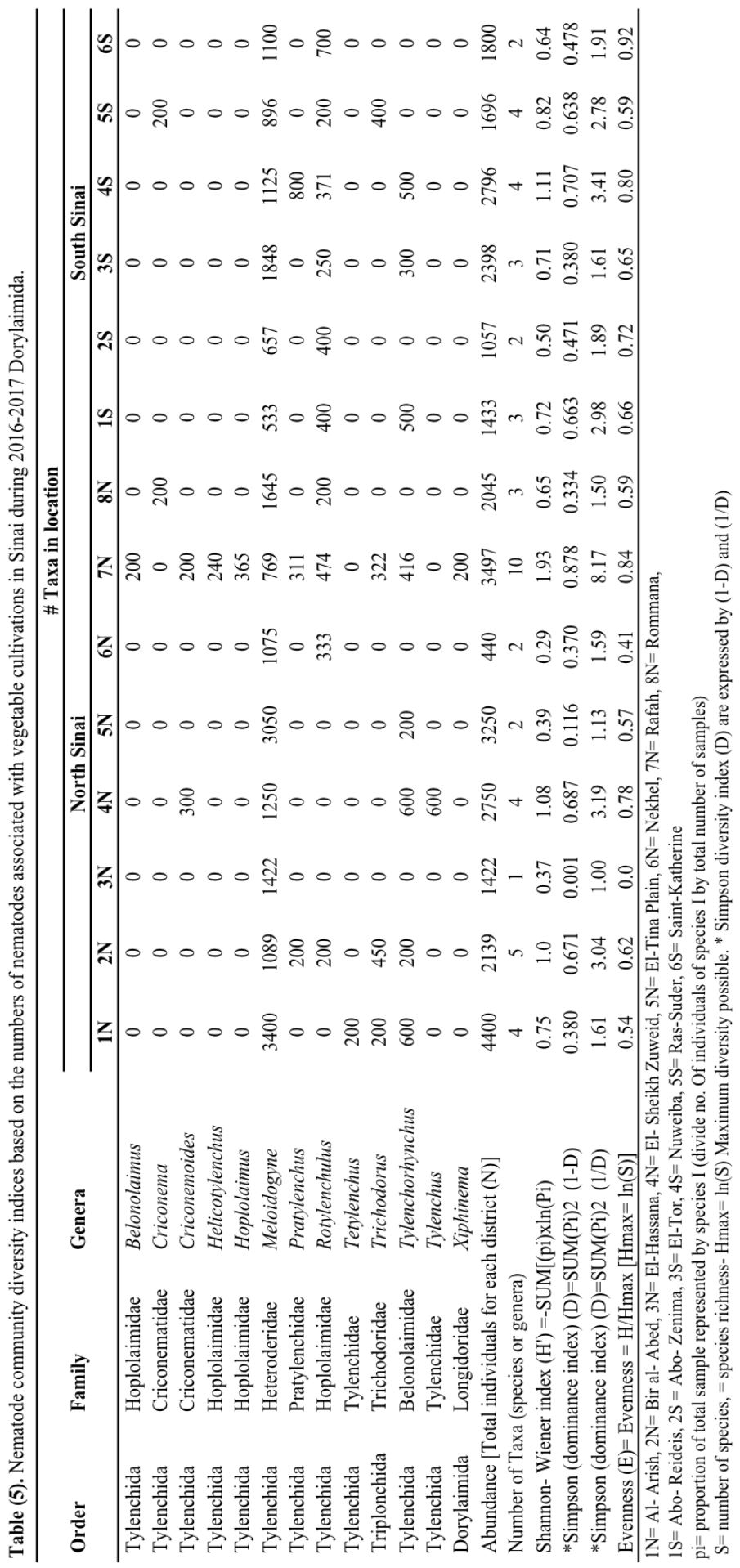

Egyptian J. Desert Res., 69, Special Issue, 77-100 (2019) 
The diversity of PPN communities, measured by numbers of nematode individuals for each species in different vegetable plantations, was compared with indices of diversity based on number of $\mathrm{H}^{\prime}$, D, beside E.H' values varied between 0.37 in El-Hassana to 1.93 in Rafah, which was considered the most diversed district in Sinai. The highest evenness value (0.84) was also found in Rafah and equalled to zero (0.0) in El-Hassana. Simpson index also peaked in Rafah in two forms (1-D) or (1/D), 0.878 and 8.15 , respectively. In South Sinai, the maximum four diversity indices were found in Newiba; 1.11, 0.707, 3.41 and 0.80 for $\mathrm{H}, \mathrm{D}-1, \mathrm{D} / 1$ and $\mathrm{E}$, respectively.

As Meloidogyne was the most abundant and dominant genus all over the surveyed Sinai surveyed locations, the sample positions were determined using GPS device as illustrated in table (6), GPS-based map was fitted to determine the hot spots of this dangerous genus with own population densities (Fig.1).

Table (6). Hot spots of root-knot nematode and their densities associated with vegetables in Sinai determined by GPS.

\begin{tabular}{cccccc}
\hline No. & N & E & PD & Density & Location \\
\hline 1 & $31^{\circ} 00^{\prime} 0.12^{\prime \prime}$ & $32^{\circ} 31^{\prime} 0.53^{\prime \prime}$ & 600 & Moderate & El-Teina $p$ \\
2 & $31^{\circ} 00^{\prime} 0.01^{\prime \prime}$ & $32^{\circ} 31^{\prime} 0.29^{\prime \prime}$ & 4300 & High & El-Teina $p$ \\
3 & $30^{\circ} 54^{\prime} 0.20^{\prime \prime}$ & $32^{\circ} 23^{\prime} 0.64^{\prime \prime}$ & 4500 & High & El-Teina $p$ \\
4 & $30^{\circ} 55^{\prime} 0.63^{\prime \prime}$ & $32^{\circ} 26^{\prime} 0.45^{\prime \prime}$ & 2800 & High & El-Teina $p$ \\
5 & $31^{\circ} 00^{\prime} 0.45^{\prime \prime}$ & $32^{\circ} 39^{\prime} 0.87^{\prime \prime}$ & 2333 & High & Rommana \\
6 & $31^{\circ} 00^{\prime} 0.09^{\prime \prime}$ & $32^{\circ} 41^{\prime} 0.14^{\prime \prime}$ & 600 & Moderate & Rommana \\
7 & $30^{\circ} 59^{\prime} 0.39^{\prime \prime}$ & $32^{\circ} 43^{\prime} 0.71^{\prime \prime}$ & 867 & Moderate & Rommana \\
8 & $31^{\circ} 00^{\prime} 0.66^{\prime \prime}$ & $32^{\circ} 34^{\prime} 0.37^{\prime \prime}$ & 200 & Low & Rommana \\
9 & $31^{\circ} 00^{\prime} 0.74^{\prime \prime}$ & $32^{\circ} 33^{\prime} 0.89^{\prime \prime}$ & 200 & Low & Rommana \\
10 & $30^{\circ} 59^{\prime} 0.48^{\prime \prime}$ & $32^{\circ} 43^{\prime} 0.83^{\prime \prime}$ & 600 & Moderate & Rommana \\
11 & $30^{\circ} 59^{\prime} 0.43^{\prime \prime}$ & $32^{\circ} 43^{\prime} 0.68^{\prime \prime}$ & 3800 & High & Rommana \\
12 & $30^{\circ} 59^{\prime} 0.43^{\prime \prime}$ & $32^{\circ} 43^{\prime} 0.71^{\prime \prime}$ & 200 & Low & Rommana \\
13 & $30^{\circ} 59^{\prime} 0.44^{\prime \prime}$ & $32^{\circ} 43^{\prime} 0.71^{\prime \prime}$ & 4200 & High & Rommana \\
14 & $30^{\circ} 59^{\prime} 0.44^{\prime \prime}$ & $32^{\circ} 43^{\prime} 0.73^{\prime \prime}$ & 600 & Moderate & Rommana \\
15 & $30^{\circ} 59^{\prime} 0.40^{\prime \prime}$ & $32^{\circ} 43^{\prime} 0.70^{\prime \prime}$ & 1000 & High & Rommana \\
16 & $30^{\circ} 59^{\prime} 0.63^{\prime \prime}$ & $32^{\circ} 55^{\prime} 0.51^{\prime \prime}$ & 467 & Moderate & Rommana \\
17 & $31^{\circ} 02^{\prime} 0.06^{\prime \prime}$ & $33^{\circ} 05^{\prime} 0.35^{\prime \prime}$ & 200 & Low & Bir Al-Abed \\
18 & $31^{\circ} 02^{\prime} 0.08^{\prime \prime}$ & $33^{\circ} 05^{\prime} 0.33^{\prime \prime}$ & 200 & Low & Bir Al-Abed \\
19 & $31^{\circ} 02^{\prime} 0.07^{\prime \prime}$ & $33^{\circ} 05^{\prime} 0.33^{\prime \prime}$ & 1000 & High & Bir Al-Abed \\
20 & $31^{\circ} 02^{\prime} 0.03^{\prime \prime}$ & $33^{\circ} 05^{\prime} 0.38^{\prime \prime}$ & 2300 & High & Bir Al-Abed
\end{tabular}


Table (6). Cont.

\begin{tabular}{|c|c|c|c|c|c|}
\hline No. & $\mathbf{N}$ & $\mathbf{E}$ & PD & Density & Location \\
\hline 21 & $31^{\circ} 02^{\prime} 0.05^{\prime \prime}$ & $33^{\circ} 04^{\prime} 0.96^{\prime \prime}$ & 700 & Moderate & Bir Al-Abed \\
\hline 22 & $31^{\circ} 02^{\prime} 0.05^{\prime \prime}$ & $33^{\circ} 04^{\prime} 0.94^{\prime \prime}$ & 600 & Moderate & Bir Al-Abed \\
\hline 23 & $31^{\circ} 00^{\prime} 0.47^{\prime \prime}$ & $32^{\circ} 56^{\prime} 0.14^{\prime \prime}$ & 3467 & High & Bir Al-Abed \\
\hline 24 & $31^{\circ} 04^{\prime} 0.53^{\prime \prime}$ & $33^{\circ} 34^{\prime} 0.00^{\prime \prime}$ & 733 & Moderate & Bir Al-Abed \\
\hline 25 & $31^{\circ} 02^{\prime} 0.51^{\prime \prime}$ & $33^{\circ} 22^{\prime} 0.74^{\prime \prime}$ & 733 & Moderate & Bir Al-Abed \\
\hline 26 & $31^{\circ} 02^{\prime} 0.52^{\prime \prime}$ & $33^{\circ} 22^{\prime} 0.74^{\prime \prime}$ & 733 & Moderate & Bir Al-Abed \\
\hline 27 & $31^{\circ} 02^{\prime} 0.53^{\prime \prime}$ & $33^{\circ} 22^{\prime} 0.71^{\prime \prime}$ & 600 & Moderate & Bir Al-Abed \\
\hline 28 & $31^{\circ} 04^{\prime} 0.30^{\prime \prime}$ & $33^{\circ} 30^{\prime} 0.61^{\prime \prime}$ & 200 & Low & Bir Al-Abed \\
\hline 29 & $31^{\circ} 07^{\prime} 0.54^{\prime \prime}$ & $33^{\circ} 48^{\prime} 0.68^{\prime \prime}$ & 533 & Moderate & Al- Arish \\
\hline 30 & $31^{\circ} 06^{\prime} 0.64^{\prime \prime}$ & $33^{\circ} 44^{\prime} 0.49^{\prime \prime}$ & 3133 & High & Al- Arish \\
\hline 31 & $31^{\circ} 06^{\prime} 0.67^{\prime \prime}$ & $33^{\circ} 44^{\prime} 0.48^{\prime \prime}$ & 4967 & High & Al- Arish \\
\hline 32 & $31^{\circ} 14^{\prime} 0.12^{\prime \prime}$ & $34^{\circ} 06^{\prime} 0.96^{\prime \prime}$ & 1175 & High & El-Sheikh Z \\
\hline 33 & $31^{\circ} 13^{\prime} 0.29^{\prime \prime}$ & $34^{\circ} 06^{\prime} 0.89^{\prime \prime}$ & 1080 & High & El-Sheikh Z \\
\hline 34 & $31^{\circ} 13^{\prime} 0.56^{\prime \prime}$ & $34^{\circ} 06^{\prime} 0.46^{\prime \prime}$ & 600 & Moderate & El-Sheikh Z \\
\hline 35 & $31^{\circ} 13^{\prime} 0.98^{\prime \prime}$ & $34^{\circ} 06^{\prime} 0.24^{\prime \prime}$ & 1300 & High & El-Sheikh Z \\
\hline 36 & $31^{\circ} 13^{\prime} 0.99^{\prime \prime}$ & $34^{\circ} 06^{\prime} 0.23^{\prime \prime}$ & 1340 & High & El-Sheikh Z \\
\hline 37 & $29^{\circ} 59^{\prime} 0.66^{\prime \prime}$ & $33^{\circ} 48^{\prime} 0.80^{\prime \prime}$ & 1167 & High & Nekhel \\
\hline 38 & $29^{\circ} 59^{\prime} 0.63^{\prime \prime}$ & $33^{\circ} 48^{\prime} 0.79^{\prime \prime}$ & 800 & Moderate & Nekhel \\
\hline 39 & $30^{\circ} 43^{\prime} 0.06^{\prime \prime}$ & $33^{\circ} 19^{\prime} 0.63^{\prime \prime}$ & 1422 & High & El-Hassana \\
\hline 40 & $29^{\circ} 36^{\prime} 22.60^{\prime \prime}$ & $32^{\circ} 43^{\prime} 21.30^{\prime \prime}$ & 350 & Low & Ras Suder \\
\hline 41 & $29^{\circ} 37^{\prime} 28.40^{\prime \prime}$ & $32^{\circ} 42^{\prime} 44.90^{\prime \prime}$ & 600 & Moderate & Ras Suder \\
\hline 42 & $29^{\circ} 37^{\prime} 36.50^{\prime \prime}$ & $32^{\circ} 42^{\prime} 46.60^{\prime \prime}$ & 850 & Moderate & Ras Suder \\
\hline 43 & $30^{\circ} 49^{\prime} 00.00^{\prime \prime}$ & $32^{\circ} 23^{\prime} 00.00^{\prime \prime}$ & 400 & Low & Ras Suder \\
\hline 44 & $31^{\circ} 18^{\prime} 07.00^{\prime \prime}$ & $34^{\circ} 11^{\prime} 59.00^{\prime \prime}$ & 987 & Moderate & Rafah \\
\hline 45 & $31^{\circ} 17^{\prime} 49.00^{\prime \prime}$ & $34^{\circ} 12^{\prime} 06.00^{\prime \prime}$ & 713 & Moderate & Rafah \\
\hline 46 & $31^{\circ} 18^{\prime} 08.00^{\prime \prime}$ & $34^{\circ} 12^{\prime} 28.00^{\prime \prime}$ & 408 & Moderate & Rafah \\
\hline 47 & $31^{\circ} 17^{\prime} 34.00^{\prime \prime}$ & $34^{\circ} 12^{\prime} 34.00^{\prime \prime}$ & 525 & Moderate & Rafah \\
\hline 48 & $31^{\circ} 17^{\prime} 35.00^{\prime \prime}$ & $34^{\circ} 11^{\prime} 46.00^{\prime \prime}$ & 756 & Moderate & Rafah \\
\hline 49 & $31^{\circ} 19^{\prime} 18.82^{\prime \prime}$ & $34^{\circ} 13^{\prime} 03.29^{\prime \prime}$ & 1267 & High & Rafah \\
\hline 50 & $31^{\circ} 18^{\prime} 49.85^{\prime \prime}$ & $34^{\circ} 12^{\prime} 25.06^{\prime \prime}$ & 960 & Moderate & Rafah \\
\hline 51 & $29^{\circ} 09^{\prime} 0.88^{\prime \prime}$ & $33^{\circ} 04^{\prime} 0.05^{\prime \prime}$ & 400 & Moderate & Abo-Zenima \\
\hline 52 & $30^{\circ} 03^{\prime} 0.51^{\prime \prime}$ & $32^{\circ} 37^{\prime} 0.62^{\prime \prime}$ & 950 & Moderate & Ras Suder \\
\hline 53 & $28^{\circ} 41^{\prime} 0.89^{\prime \prime}$ & $33^{\circ} 40^{\prime} 0.72^{\prime \prime}$ & 500 & Moderate & El-Tor \\
\hline
\end{tabular}


Table (6). Cont.

\begin{tabular}{|c|c|c|c|c|c|}
\hline No & $\mathbf{N}$ & $\mathbf{E}$ & PD & Density & Location \\
\hline 54 & $28^{\circ} 41^{\prime} 0.89^{\prime \prime}$ & $33^{\circ} 40^{\prime} 0.73^{\prime \prime}$ & 550 & Moderate & El-Tor \\
\hline 55 & $28^{\circ} 16^{\prime} 0.86^{\prime \prime}$ & $33^{\circ} 37^{\prime} 0.31^{\prime \prime}$ & 2900 & High & El-Tor \\
\hline 56 & $28^{\circ} 16^{\prime} 0.84^{\prime \prime}$ & $33^{\circ} 37^{\prime} 0.32^{\prime \prime}$ & 900 & Moderate & El-Tor \\
\hline 57 & $28^{\circ} 17^{\prime} 0.33^{\prime \prime}$ & $33^{\circ} 37^{\prime} 0.41^{\prime \prime}$ & 650 & Moderate & El-Tor \\
\hline 58 & $28^{\circ} 17^{\prime} 0.33^{\prime \prime}$ & $33^{\circ} 37^{\prime} 0.41^{\prime \prime}$ & 200 & Low & El-Tor \\
\hline 59 & $28^{\circ} 20^{\prime} 0.85^{\prime \prime}$ & $33^{\circ} 38^{\prime} 0.48^{\prime \prime}$ & 4900 & High & El-Tor \\
\hline 60 & $28^{\circ} 17^{\prime} 0.99^{\prime \prime}$ & $33^{\circ} 35^{\prime} 0.76^{\prime \prime}$ & 1700 & High & El-Tor \\
\hline 61 & $28^{\circ} 19^{\prime} 0.85^{\prime \prime}$ & $33^{\circ} 38^{\prime} 0.77^{\prime \prime}$ & 1533 & High & El-Tor \\
\hline 62 & $28^{\circ} 43^{\prime} 0.00^{\prime \prime}$ & $34^{\circ} 06^{\prime} 0.53^{\prime \prime}$ & 1533 & High & Siant Kath. \\
\hline 63 & $28^{\circ} 40^{\prime} 0.66^{\prime \prime}$ & $33^{\circ} 58^{\prime} 0.86^{\prime \prime}$ & 2200 & High & Siant Kath. \\
\hline 64 & $28^{\circ} 41^{\prime} 0.70^{\prime \prime}$ & $33^{\circ} 56^{\prime} 0.85^{\prime \prime}$ & 200 & Low & Siant Kath. \\
\hline 65 & $28^{\circ} 55^{\prime} 0.66^{\prime \prime}$ & $34^{\circ} 35^{\prime} 0.76^{\prime \prime}$ & 350 & Low & Newiba \\
\hline 66 & $28^{\circ} 31^{\prime} 0.60^{\prime \prime}$ & $34^{\circ} 28^{\prime} 0.17^{\prime \prime}$ & 800 & Moderate & Newiba \\
\hline 67 & $28^{\circ} 15^{\prime} 0.24^{\prime \prime}$ & $33^{\circ} 36^{\prime} 0.79^{\prime \prime}$ & 800 & Moderate & El-Tor \\
\hline 68 & $28^{\circ} 42^{\prime} 0.51^{\prime \prime}$ & $33^{\circ} 46^{\prime} 0.16^{\prime \prime}$ & 200 & Low & WadiFiran \\
\hline 69 & $28^{\circ} 41^{\prime} 0.92^{\prime \prime}$ & $33^{\circ} 39^{\prime} 0.25^{\prime \prime}$ & 200 & Low & WadiFiran \\
\hline 70 & $30^{\circ} 40^{\prime} 44.07^{\prime \prime}$ & $33^{\circ} 30^{\prime} 35.88^{\prime \prime}$ & 200 & Low & El-Maghara \\
\hline 71 & $30^{\circ} 35^{\prime} 26.71^{\prime \prime}$ & $33^{\circ} 22^{\prime} 17.70^{\prime \prime}$ & 400 & Moderate & El-Maghara \\
\hline 72 & $30^{\circ} 27^{\prime} 59.78^{\prime \prime}$ & $33^{\circ} 47^{\prime} 3.25^{\prime \prime}$ & 200 & Low & El-Maghara \\
\hline 73 & $30^{\circ} 28^{\prime} 12.70^{\prime \prime}$ & $33^{\circ} 47^{\prime} 0.94^{\prime \prime}$ & 600 & Moderate & El-Maghara \\
\hline 74 & $29^{\circ} 54^{\prime} 12.54^{\prime \prime}$ & $33^{\circ} 44^{\prime} 3.64^{\prime \prime}$ & 300 & Low & Nekhel \\
\hline 75 & $29^{\circ} 53^{\prime} 23.51^{\prime \prime}$ & $33^{\circ} 42^{\prime} 14.75^{\prime \prime}$ & 200 & Low & Nekhel \\
\hline
\end{tabular}

$\mathrm{PD}=$ Population density, $\mathrm{N}=$ latitude, $\mathrm{E}=$ longitude 


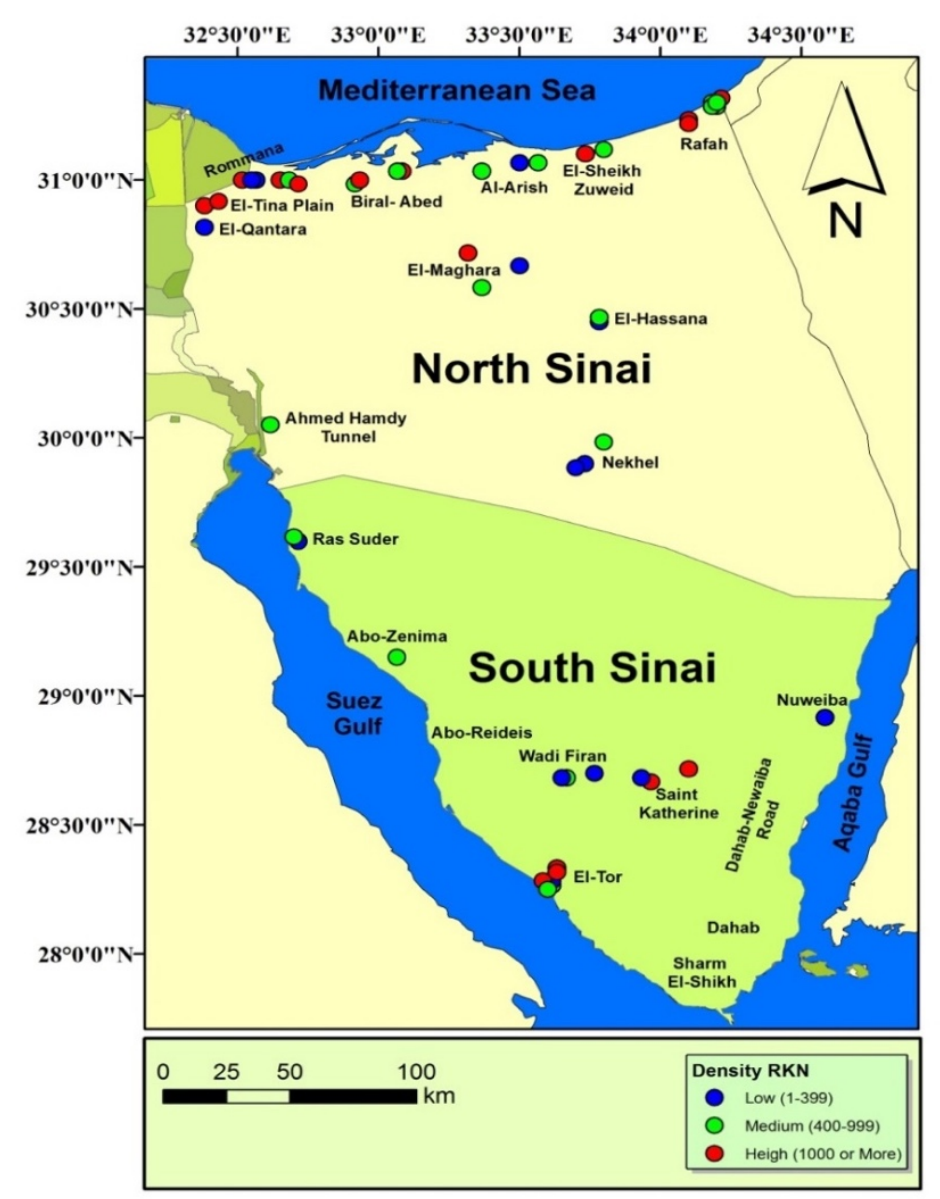

Fig. (1). GPS based map showed distribution of root-knot nematodes associated with vegetables growing in Sinai Peninsula.

\section{DISCUSSION}

Phytoparasitic nematodes infect almost all-important agronomical crops, also they constitute a major challenge to the production of many crops, especially in light or sandy soil and newly reclaimed desert lands (Ibrahim et al., 2010). Current survey revealed that thirteen stylet bearing nematode genera were encountered in soil samples collected from different vegetable cultivations in Sinai Peninsula, including ectoparasites (Belonolaimus, Criconema, Criconemoides, Helicotylenchus, Hoploliamus, Tetylenchus, Trichodorus, Tylenchorhynchus, Tylenchus and Xiphinema), endoparasites (Meloidogyne and Pratylenchus) and semi-endoparasites (Rotylenchulus). 
Presence of these serious phytoparastic nematodes on vegetables cultivations must be taken seriously by growers, especially they live in warmed areas. The association of these nematodes with vegetable crops has been reported to diminish yields (Anwar and McKenry, 2012). Many investigations were carried out to analyze the parasitic nematode community in several vegetable fields. Another investigator found that seven PPN genera associated with vegetable crops viz. Helicotylenchus, Meloidogyne, Pratylenchus, Rotylenchus, Scutellonema, Quinisulcius, and Tylenchus in Uganda (Bafokuzara, 1996).

Meloidogyne is by far the most dominant PPN both in North and South Sinai governorates. This widespread distribution of Meloidogyne in all agricultural regions is consistent with results from earlier surveys (Ibrahim, 1994; Ibrahim et al., 2000; Korayem and Mohamed, 2010; Korayem et al., 2014). The global distribution of Meloidogyne compared with the rest nematode genera, especially in tropical, subtropical and Mediterranean climates, are due to wide and various host ranges that lacking specificity in parasitism (Sasser, 1979 and Anwar et al., 2006). The status of vegetable crops as a good host for Meloidogyne has been previously reported (Siddiqi, 2000; Sikora and Fernandez, 2005; Kaskavalci, 2007; Baimey et al., 2009; Chaudhary et al., 2011; Anwar et al., 2013 and Rani, 2017) and this was also supported by the present survey.

All nematode genera found in South Sinai (Criconema, Meloidogyne, Pratylenchus, Rotylenchulus, Trichodorus and Tylenchorhynchus) were firstly recorded in this governorate, as this is the first nematological survey carried out in South Sinai. Seven genera viz., Belonolaimus, Criconema, Criconemoides, Helicotylenchus, Pratylenchus, Rotylenchulus, and Tetylenchus also were not reported before the present survey in North Sinai associating with vegetables, whereas Ashoub (2010) reported that only Meloidogyne genus associated with vegetable plants., and in the previous survey conducted by Korayem et al. (2014), they found 6 genera (Hoplolaimus, Meloidogyne, Trichodorus,Tylenchorhynchus, Tylenchus and Xiphinema) in some North Sinai locations.

In general, the total number of PPN in each district ranged from 1408 (Nekhel) to 3250 (El-Tina Plain) individuals $/ 250-\mathrm{cm}^{3}$ soil and in South Sinai from 1075 (Abo-Zenima) to 2796 (Nuweiba). It was found that the highest occurrence was possessed by Meloidogyne (44.8\%) and the lowest was achieved by Belonolaimus, Criconema and Xiphinema (0.25\%) in North Sinai. Meloidogyne also was occupying the top position in South Sinai as its repetition was $34.5 \%$, while the least frequency $(0.5 \%)$ was recorded by Criconema and Pratylenchus. Interestingly, Meloidogyne, which considered the most damaging genus was detected in all surveyed districts in all Sinai Peninsula governorates. The second repetition rate (7\%) in North Sinai was owned by Rotylenchulus as it was recovered from four districts only, but it found in all districts of South Sinai with frequency of $10.3 \%$.

Egyptian J. Desert Res., 69, Special Issue, 77-100 (2019) 
The diversity indices, Shannon-Wiener's H', 1-D\& 1/D (inverse of Simpson's index) were calculated based on the abundance and frequency of each nematode genus. The 1/D was used to yield larger values for more diverse communities, as well as $\mathrm{H}^{\prime}$ beside $\mathrm{E}$ (Evenness). The diversity and spatial patterns of PPN vary according to many factors like; environmental and behavioral factors, plant species, cultivation intensity, monoculture of local varieties, irrigation system and soil type as previously mentioned (Baimey et al., 2009). Also, the probability of species detection is influenced by population density and spatial dispersion properties (Prot and Ferris, 1992). The present results indicated that PPN populations and diversity may increase with higher rainfall and continuous cultivation more than effect of water content and temperature on PPN increases (Govaerts et al., 2007). Earlier findings stated that abundance and population of some nematode species were associated with particular specific soil textures (Jones et al., 1969 and Bongers, 1988). It is also known that populations of some of the nematodes were markedly reduced by soil cultivation and cropping systems (Oostenbrink, 1964 and Jones et al., 1969). It was previously mentioned that soil physico-chemical properties, climate and agricultural practices have a great impact on the diversity of PPN communities. The interaction between phytoparasitic nematode communities and edaphic factors are poorly understood in most agrosystems (Ortiz et al., 2010; Duyck et al., 2012; Godefroid et al., 2013; Kavitha and Vanita Das, 2015 and Godefroid et al., 2017). Organic matter and previous crops can favor the reproduction of certain nematode species. Godefroid et al. (2017) recorded that Pratylenchus coffeae was more abundant in regions have a high exchangeable cation content and low organic matter content. Nematode abundances were apparently affected by the previous crops e.g., Radopholus similis was particularly abundant in fields where banana or plantain were the previous crops; and Meloidogyne were abundant in fields where a market garden was the previous crop. Kumar et al. (2017) observed that sandy loam type favor reproduction of RKN. In contrary, Baimey et al. (2009) found a weak relationship between soil physico-chemical properties and nematode population density.

However, cultivation on Sinai Peninsula, especially in the South Sinai depends on water free of nematodes (rain and wells), vice versa; in El-Salam canal, in the north, which feeds El-Tiena plain by irrigated water, helps to distribute nematodes from a place to another and encourage the farmers to cultivate the soil many times yearly. Recent investigations found significant possibilities for increased nematode diversity and greater prevalence of both Meloidogyne and Pratylenchus with increasing rainfall. Also, climate change, especially temperature. These findings suggest that significant changes in nematode populations were linked to changing climate, cropping systems and agriculture practices (Fleming et al., 2016 and Hamza et al., 2018).

So, these results are beneficial for future planning for managing PPN in Sinai and protect new targeted plantations from plant parasitic nematodes, 
in particular root knot nematode (RKN) Meloidogyne as its hot spots were determined in vegetable cultivation in Sinai Peninsula (Fig. 1). These finding are in harmony with previous finding (Gautam et al., 2014; Korayem et al., 2014; Singh and Khanna, 2015; Myint et al., 2017 and Rani, 2017). In these areas showing severe infestation, especially for the most dangerous nematode (Meloidogyne), root-lesion nematode (Pratylenchus) and for ectoparasitic genera, which considered a viral vectors (Trichodorus and Xiphinema), it is advisable to adapt strict integrated disease management strategies for controlling these nematodes. In view of damage potential of these hidden enemies of plants, an urgent need is felt for commencing coordinated efforts at national and regional level pertaining to distribution and dissemination of plant parasitic nematodes, estimating of crop losses and for developing nematode management strategies. Observations and results obtained from this survey clarify that host plants vary in their susceptibility to PPN infection, so cropping sequence may play an important role in phytonematodes management and reducing nematicides use.

\section{CONCLUSION}

The present study represents the first survey in South Sinai and one of the few surveys carried out in North Sinai. It was found that 13 plant parasitic nematode (PPN) genera variably distributed in different surveyed localities of vegetable cultivations in Sinai Peninsula. The most predominant genus was the Meloidogyne, which was found in all locations under this extensive survey, the frequency of this nematode was varied from district to other. The diversity of nematode fauna was found to be affected by many factors including; plant host, cultivation intensity, soil type, irrigation system and. Current and future studies should put the objective of mapping out of plant parasitic nematodes incidence and impact of cropping sequence in survival, diversity and dynamics of PPN. Finally, any development program on susceptible vegetable plants in these infested areas should include control measures against these nematodes, so this study can be useful for the design of phytoparasitic control programs in vegetable fields.

\section{ACKNOWLEDGEMENT}

The authors would like to thank the Prof. Dr. Ismail Abdelgalil, previous president of Desert Research Center (DRC) and Prof. Dr. Naiim Moselhy, current president of DRC for their continuous support. Special thanks are devoted to Mr. Sayed Abdalla for his sincere help in drawing maps.

Egyptian J. Desert Res., 69, Special Issue, 77-100 (2019) 


\section{REFERENCES}

Adamou, H., B. Adamou, H.A. Oumarou, S. Ramatou, D. Seyni and E. Toudou (2013a). Characterization of plant-parasitic nematode communities associated with tomato, eggplant and pepper in the suburban area of Niamey (Niger). Int. J. Agri. Crop. Sci., 5 (20): 2488-2494.

Adamou, H., S. Etienne and D. Ramatou (2013b). Biodiversity of plantparasitic nematodes associated with pepper in the regions of Diffa and Dosso (Niger republic). Int. J. Farm. Alli. Sci., 2 (15): 482-487.

Anderson, R.V. and R.H. Mulvey (1979). Plant-parasitic nematodes in Canada Part 1. An illustrated key to the genera. Biosystematics Research Institute, Ottawa, Ontario, Monograph/Research Branch, Agriculture, Canada No. 20.

Anwar, A.S., M.M. Mahdi, M.V. McKenry and A. Qadir (2013). Survey of plant-parasitic nematodes associated with four vegetable crops cultivated within tunnels. Pak. J. Zool., 45 (3): 595-603.

Anwar, A.S., M.V. McKenry and N. Javad (2006). The root-knot nematodes: destructive M.C. pests of crops. Proc. Int. Symp. Sust. Crop Impro. Integ. Manag. Organized by Fac. Agric., Univ. Agric., Faisalabad, Pakistan, September 14-16, p. 216-222.

Anwar, A.S. and M.V. McKenry (2012). Incidence and population density of plant-parasitic nematodes infecting vegetable crops and associated yield losses in Punjab, Pakistan. Pak. J. Zool., 44 (2): 327-333.

Ashoub, A.H. (2010). Community analysis of plant parasitic nematode in North Sinai. Egypt. J. Agronematol., 9 (2): 91-102.

Bafokuzara, D.N. (1996). Incidence of different nematodes on vegetable and fruit crops and preliminary assessment of yield loss due to Meloidogyne species in Uganda. Nematol. Bras., 20 (1): 32-44.

Baimey, H., D. Coyneb, G. Dagbenonbakinc and B. Jamesa (2009). Plantparasitic nematodes associated with vegetable crops in Benin: Relationship with soil physio-chemical prosperities. Nematol. Medit., 37: $227-236$.

Becker, J.O. (2014). Plant Health Management: Crop Protection with Nematicides. In: 'Encyclopedia of Agriculture and Food Systems'. Elsevier, London, p. 400-407.

Bongers, T. (1988). In "De Nematoden van Nederland". Natuurhistorische Bibliotheek KNNV. Nr. 46. Pirola, Schoorl The Netherlands.

Boyle, T.P., G.M. Smillie, J.C. Anderson and D.R. Beeson (1990). A sensitivity analysis of nine diversity and seven similarity indexes. Res. J. Water Poll. Control, 62: 749-762.

Chaudhary, K.K., D. Brhane, H. Okube, T. Zaid and E. Dagnew (2011). Distribution, frequency of occurrence and population density of root 
knot nematode in Hamelmalo - Eritrea, Afr. J. Microbiol. Res., 5 (31): 5656-5661.

Cobb, N.A. (1918). Estimating the nematode population of the soil. [Washington]: United States Department of Agriculture, 1918. 48 pp. (Agricultural Technical Circular, 1).

Duyck, P.F., E. Dortel, P. Tixier, F. Vinatier, P.M. Loubana, C. Chabrier and P. Quénéhervé (2012). Niche partitioning based on soil type and climate at the landscape scale in a community of plant-feeding nematodes. Soil Biol. Biochem., 44: 49-55.

Fleming, T.R., N.E. McGowan, A.G. Maule and C.C. Fleming (2016). Prevalence and diversity of plant parasitic nematodes in Northern Ireland grassland and cereals, and the influence of soils and rainfall. Plant Pathol., 65: 1539-1550.

Gautam, S.K., G. Sahu, B.K. Verma and A.N. Poddar (2014). Status of rootknot nematode (Meloidogyne species) disease in vegetable crops of some districts of central plain region of Chhattisgarh state. India. Afr. J. Microbiol. Res., 8 (16): 1663-1671.

Godefroid, M., L. Delaville, S. Marie-Luce and P. Quénéhervé (2013). Spatial stability of a plant-feeding nematode community in relation to macroscale soil properties. Soil Biol. Biochem., 57: 173 -181.

Godefroid, M., P. Tixierb, C. Chabrierb, D. Djigal and P. Quénéhervé (2017). Associations of soil type and previous crop with plant-feeding nematode communities in plantain agrosystems. Appl. Soil Ecol., 113: 63-70.

Goodey, J.B. (1963). In: 'Soil and Fresh-Water Nematodes'. New York: John Wiley and Sons.

Govaerts, B., M. Fuentes and M. Mezzalama (2007). Infiltration, soil moisture, root rot and nematode populations after 12 years of different tillage, residue and crop rotation managements. Soil and Tillage Research, 94: 209-19

Hamza, M.A., A. Moukhli, Z. Ferji, O. Fossati-Gaschignard, J. Tavoillot, N. Ali, H. Boubaker, A. El Mousadik and T. Mateille (2018). Diversity of plantparasitic nematode communities associated with olive nurseries in Morocco: Origin and environmental impacts. Appl. Soil Ecol., 124: 7-16.

Ibrahim, I.K.A. (1994). Potentially important phytoparasitic nematodes in agroforesty plantations and the associated host plants. Proc. of the $2^{\text {nd }}$ Afro-Asian Nematol. Symp., Menoufia, Egypt.

Ibrahim, I.K.A. and T.A. El-Sharkawy (2001). Genera and species of phytoparasitic nematodes and the associated host plants in Egypt. Adv. Agric. Res. Egypt, 3 (1): 75-95.

Ibrahim, I.K.A., Z.A. Handoo and A.A. El-Sherbiny (2000). A survey of phytoparasitic nematodes on cultivated and non-cultivated plants in Northwestern Egypt. J. Nematol., 32 (4S): 478-485.

Egyptian J. Desert Res., 69, Special Issue, 77-100 (2019) 
Ibrahim, I.K.A., A.A. Mokbel and Z.A. Handoo (2010). Current status of phytoparasitic nematodes and their host plants in Egypt. Nematropica, 40 (2): 239-262.

Jones, F.G.W., D.W. Larbey and D.M. Parrott (1969). The influence of soil structure and moisture on nematodes, especially Xiphinema, Longidorus, Trichodorus, and Heterodera spp. Soil Biol. Biochem., 1: $153-165$.

Kaskavalci, G. (2007). Effect of soil solarization and organic amendment treatment for controlling Meloidogyne incognita in tomato cultivars in Western Anatolia. Turk. J. Agric. For., 31: 159-167.

Kavitha, B. and V. Vanita Das (2015). Characterization of plant-parasitic nematode communities associated with brinjal crops in the Nagole Area of Rangareddy District, Hyderabad, Telangana, India (2011\&2012). Int. J. Eng. Sci. Res., 5: 2297-2293.

Korayem, A.M. and M.M.M. Mohamed (2010). High yielding hybrid maize cultivar tolerant to Pratylenchus zeae in Egypt. Pak. J. Nematol. 28 (1): 109-114.

Korayem., A.M., M.M.A. Youssef, M.M.M. Mohamed and A.M.S. Lashein (2014). A survey of plant parasitic nematodes associated with different plants in North Sinai. Middle East J. Agric. Res., 3 (3): 522529.

Kumar, S., C.B. Yadav and A.A. Tiwari (2017). Survey of root-knot nematodes associated with tomato crops in Fatehabad, Agra. Indian Res. J. Gene. Biotechnol., 9 (1): 73-77.

Kvalseth, T.O. (1991). Note on biological diversity, evenness, and homogeneity. Oikos, 62 (1): 123-127.

Mai, W.F. and H.H. Lyon (1975). In: 'Pictorial Key to Genera of PlantParasitic Nematodes'. Cornell University Press, Ithaca and London, NY, No. $4^{\text {th }}$ revised, 219 pp.

Mekete, T., A. Dababat, N. Sekora, F. Akyazi and E. Abebe (2012). Identification key for agriculturally important plant-parasitic nematodes. Prepared for the International Nematode Diagnosis and Identification Course 2012 - A manual for nematology. Mexico, D.F.: CIMMYT.

Mokbel, A.A, I.K.A. Ibrahim, M.A.M. El-Saedy and S.E. Hammad (2006). Plant parasitic nematodes associated with some fruit trees and vegetable crops in northern Egypt. Egypt. J. Phytopathol., 34: 43-51.

Myint, N.N., Y.Y. Aye, Y.Y. Aung and S. Bawm (2017). Detection of plant parasitic nematodes in the soil of crop field in Meiktila area, Myanmar. J. Agric. Sci.,1: 258-263.

Norton, D.C. (1978). In: 'Ecology of Plant Parasitic Nematodes'. John Wiley and Sons, New York, 268 pp.

Oostenbrink, M. (1964). Harmonious control of nematode infestation. Nematologica, 10: 49-56. 
Ortiz, B.V., C. Perry, P. Goovaerts, G. Vellidis and D. Sullivan (2010). Geostatistical modeling of the spatial variability and risk areas of southern root-knot nematodes in relation to soil properties. Geoderma, 156: 243-252.

Oteifa, B.A., M.M. Shams Eldean and M.H. El Hamawy (1997). A preliminary compiled study on the biodiversity of free-living, plantand insect-parasitic nematodes in Egypt. Egypt. J. Agronematol., 1: $1-36$.

Peet, R.K. (1974). The measurement of species diversity. Annu. Rev. Ecol. Syst., 5: 285-307.

Pielou, E.C. (1975). In: “Ecological Diversity”. Wiley, New York, 165 p.

Prot, J.C. and H. Ferris (1992). Sampling approaches for extensive surveys in nematology. J. Nematol., 24 (S): 757-764.

Rani, V.P. (2017). Identification of root-knot nematodes in the selected agro ecosystems of Telangana state. Int. J. Zool. Res., 7: 1-6.

Sasser, J.N. (1979). Economic importance of Meloidogyne in tropical countries. In: Lamberti F. and C.E. Taylor (eds). 'Root-knot Nematodes (Meloidogyne. Species): Systematics, Biology and Control'. Academic Press, London, p. 359-374.

Sawadogo, A., B. Thio, S. Kiemde, I. Drabo, C. Dabire, J. Ouedraogo, T.R. Mullens, J.D. Ehlersand and P.A. Roberts (2009). Distribution and prevalence of parasitic nematodes of cowpea (Vigna unguiculata) in Burkina Faso. J. Nematol., 41: 120-127.

Shakeel, Q., N. Javed, Y. Iftikhar, I.U. Haq, S.A. Khan and Z. Ullah (2012). Association of plant parasitic nematode with four vegetable crops. Pak. J. Phytopathol., 24 (2): 143-148.

Shannon, C.E. and W. Wiener (1949). In: 'The Mathematical Theory of Communication'. University of Illinois Press, Urbana. $117 \mathrm{pp}$.

Siddiqi, M.R. (2000). In: 'Tylenchida Parasites of Plants and Insects'. Second Ed. CAB International, Wallingford, UK, $848 \mathrm{pp}$.

Sikora, R.A and E. Fernandez (2005). Nematode parasites of vegetables. In: Luc et al. (eds.). 'Plant Parasitic Nematodes in Subtropical and Tropical Agriculture'. $2^{\text {nd }}$ edition, CAB International, Wallingford, UK, p. 319-392.

Simpson, E. (1949). Measurements of diversity. Nature, 163: 688.

Singh, P. and A. Khanna (2015). Incidence of phytoparasitic nematodes in vegetable crops grown under protected cultivation in Himachal Pradesh. Int. J. Sci. Environ. Technol., 4 (6): 1640-1646.

Tariq-Khan, M., A. Munir, T. Mukhtar, J. Hallmann and H. Heuer (2017). Distribution of root-knot nematode species and their virulence on vegetables in northern temperate agro-ecosystems of the Pakistaniadministered territories of Azad Jammu and Kashmir. J. Plant Dis. Prot., 124 (3): 201-212.

Egyptian J. Desert Res., 69, Special Issue, 77-100 (2019) 
Tarjan, A.C. (1973). A synopsis of the genera and species in the Tylenchorhynchinae (Tylenchoidea, Nematoda). Proc. Helminthol. Soc. Wash., 40: 123-144.

Vindhyarani, P. (2017). Species diversity of phytophagous nematodes in selected agroecosystems of Telangana State. Int. J. Zool. Stud., 2: 14.

Yeates, G.W. and T. Bongers (1999). Nematode diversity in agroecosystems. Agriculture, Ecosystems and Environment, 74: 113-135. 


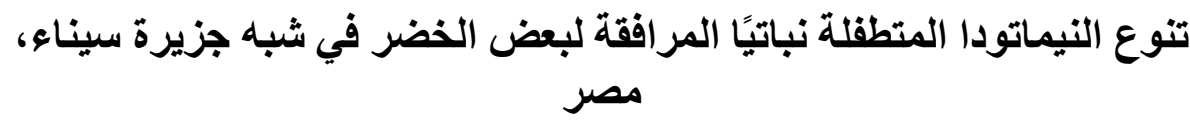

أحمد سليمان محمد محمد النوبي'“، السيد عبد العزيز منتصرَ وإسماعيل عبد الله 'قسم وقاية النبات، مركز بحوث الصحر اءو، القاهرة "قسم الحيو ان الزر اعي و النيماتودا، كلية الزر اعة، جامعة الأزهر

تم إجر اء مسح للنيماتودا المنطفلة نباتيًا في زراعات الخضر في شبه فئه جزيرة سيناء، حيث

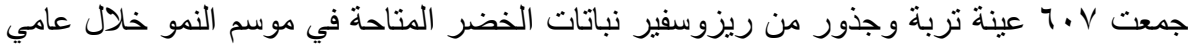

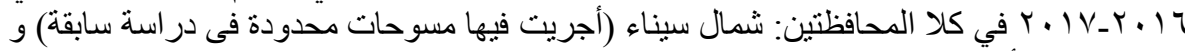

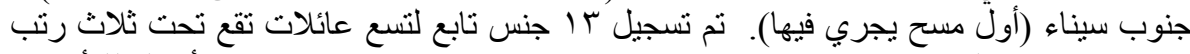

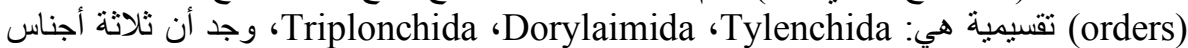

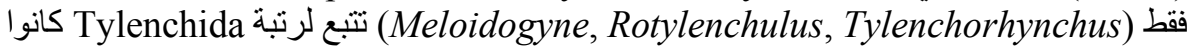

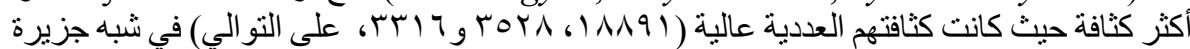

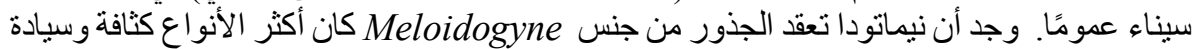

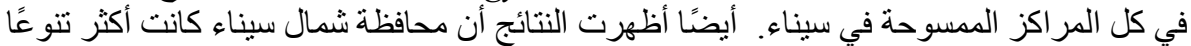

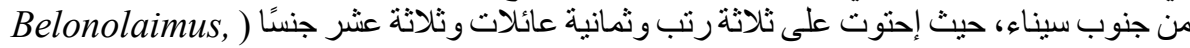
Criconema, Criconemoides, Helicotylenchus Hoplolaimus, Meloidogyne, Pratylenchus, Rotylenchulus, Tetylenchus, Trichodorus, Tylenchorhynchus, (Tylenchusandxiphinema Criconema, Meloidogyne, Pratylenchus, Rotylenchulus, ) (TrichodorusandTylenchorhynchus

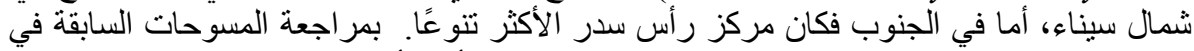

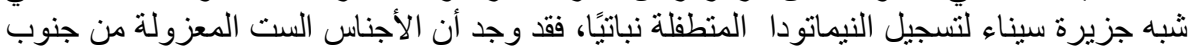

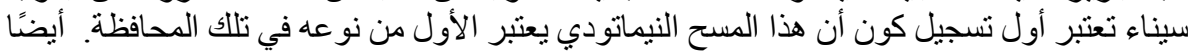
هذه الدر اسة سوف تلفت إنتباه المز ارعين للعمل على مكافحة التلوث بالنيماتودا. 